\title{
Recomendaciones para la atención de pacientes con diabetes mellitus con factores de riesgo o enfermedad cardiovascular establecida y SARS-CoV-2
}

\section{Recommendations for the care of patients with diabetes mellitus with risk factors or established cardiovascular disease and SARS-CoV-2}

Marco A. Alcocer-Gamba ${ }^{1 *}$, Pedro Gutiérrez-Fajardo ${ }^{2}$, Alejandro Sosa-Caballero ${ }^{3}$, Alfredo Cabrera-Rayo ${ }^{4}$, Raquel N. Faradji-Hazan³, Francisco G. Padilla-Padilla1,2, Juan C. Garnica-Cuellar ${ }^{3}$, Leticia M. Hernández-Arispe ${ }^{3}$, Fernando A. Reyes-Cianeros ${ }^{1,2}$, Andrés León-Suárez ${ }^{3}$, José de-JesúsRivera $^{1,2}$, Leonardo Mancillas-Adame ${ }^{3}$, Manuel Gaxiola-Macias ${ }^{1,2}$, Eduardo Márquez-Rodríguez ${ }^{3}$, Emma Miranda-Malpica', Valentín Sanchez-Pedraza ${ }^{3}$, Daniel S. Lara-Martínez', Antonio Segovia-Palomo ${ }^{3}$, Angeles Nava-Hernández ${ }^{1}$ y Romina Rivera-Reyes ${ }^{1}$

${ }^{1}$ Sociedad Mexicana de Cardiología; ${ }^{2}$ Asociación Nacional de Cardiólogos de México; ${ }^{3}$ Sociedad Mexicana de Nutrición y Endocrinología; ${ }^{4}$ Colegio de Medicina Interna de México. Ciudad de México, México

\section{Resumen}

Se realiza una revisión sobre el riesgo de los pacientes que padecen diabetes mellitus en el contexto de morbimortalidad general y relacionada a infección por el coronavirus 2 del síndrome respiratorio agudo grave (SARS-CoV-2). Así mismo se repasan las recomendaciones generales, de alimentación y de la prevención de las comorbilidades que más frecuentemente padecen dichos enfermos. Finalmente se hace una revisión de las recomendaciones farmacológicas sobre el tratamiento tanto oral como parenteral en el paciente ambulatorio, en la hospitalización y en estados críticos infectados por el SARS-CoV-2.

Palabras clave: Diabetes Mellitus tipo 2. COVID-19. Tratamiento. Enfermedad cardiovascular. Factores de riesgo. SARS-CoV-2.

\begin{abstract}
A review is carried out to examine the risk of patients suffering from diabetes mellitus in the context of general morbidity and mortality and related to infection by SARS-CoV-2. Likewise, the general recommendations for food and the prevention of comorbidities that most these patients suffer most frequently are also studied. Finally, a review of the pharmacological recommendations on both oral and parenteral treatment in the outpatient, in hospitalization and in critical states infected with SARS-CoV-2 is made.
\end{abstract}

Key words: Type 2 diabetes mellitus. COVID-19. Cardiovascular disease treatment. Risk factors. SARS-CoV-2.

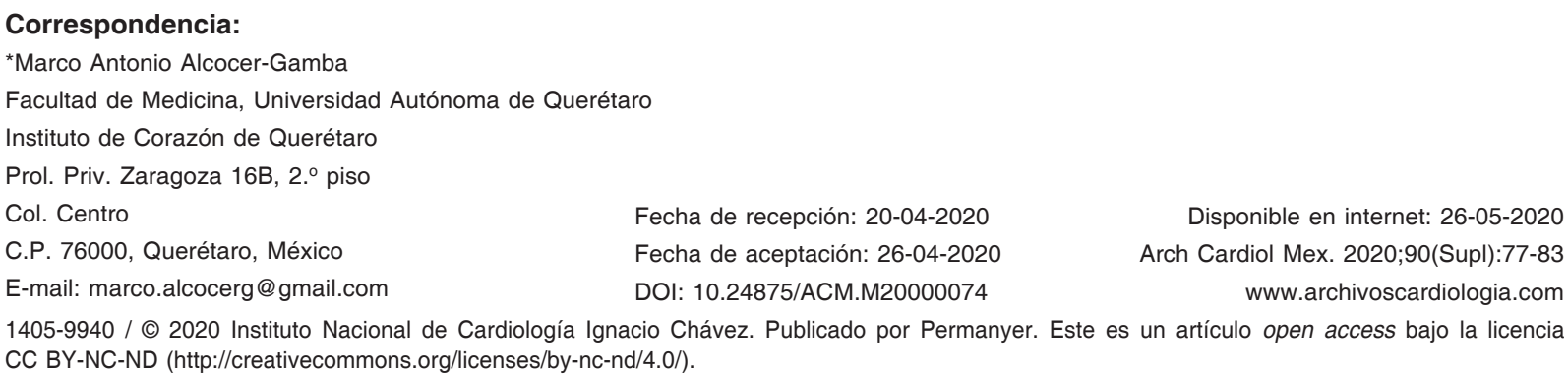

Fecha de recepción: 20-04-2020 Fecha de aceptación: 26-04-2020 DOI: 10.24875/ACM.M20000074

Disponible en internet: 26-05-2020 Arch Cardiol Mex. 2020;90(Supl):77-83 www.archivoscardiologia.com 1405-9940 / ๑ 2020 Instituto Nacional de Cardiología Ignacio Chávez. Publicado por Permanyer. Este es un artículo open access bajo la licencia CC BY-NC-ND (http://creativecommons.org/licenses/by-nc-nd/4.0/). 


\section{Introducción}

Entre el 5 y el $7 \%$ de las personas con coronavirus 2 del síndrome respiratorio agudo grave (SARS-CoV-2) tiene diabetes mellitus (DM), aunque pueden llegar hasta el $50 \%$ los que presentan hiperglucemia durante el evento infeccioso'. Esta asociación incrementa los riesgos de en al menos el doble que para la población sin comorbilidades ${ }^{2,3}$ y alrededor del $10 \%$ requerirán manejo intensivo por la presentación severa de SARS-CoV-2. Más del $20 \%$ de las personas que fallecen por SARS-CoV-2 padecen DM². El incremento de riesgo está asociado a mayor susceptibilidad a infecciones e incremento de la mortalidad ante estas, aunque en la actualidad es un punto de discusión ${ }^{4}$.

Evidencia más consistente muestra que niveles de hemoglobina glucosilada A1c ( $\mathrm{HbA1c}$ ) arriba del 9\% aumentan el $60 \%$ de hospitalización por neumonía grave en personas con $\mathrm{DM}^{5}$. Además, la tasa de mortalidad con DM descontrolada puede variar del 22 al $31 \%{ }^{6,7}$. Esto significa un mayor riesgo debido a que la población mexicana tiene bajos niveles de control glucémico con base en la $\mathrm{HbA1c}$, estimando que solo el $25 \%$ de las personas que viven con diabetes se encuentran en metas; hay además pobre frecuencia de medición de HbA1c, ya que solo el $15 \%$ de la población se realiza el estudio una sola vez al año ${ }^{8}$.

\section{Diabetes mellitus y SARS-CoV-2}

Está fuertemente establecida la vinculación entre control y estabilidad glucémica con el pronóstico evolutivo de la infección por SARS-CoV-29,10. El SARSCoV-2 se une a los islotes de Langerhans por el receptor de la enzima convertidora de angiotensina (ECA) 2, los destruye y favorece la progresión de hiperglucemia. Por otro lado, también favorece un estado inflamatorio con el incremento de la interleucina (IL) 6 y proteína $C$ reactiva, un estado proinflamatorio ya conocido que se prolonga en las personas con diabetes; así mismo, es un estado procoagulante con aumento en las concentraciones de dímero $D$, así como la asociación a una inadecuada regulación del sistema inmunitario ${ }^{11-14}$. Es conocido que la hiperglucemia crónica, la hiperglucemia aguda, la hipoglucemia y la alta variabilidad glucémica son estados que se vinculan con sobreexpresión de citocinas, y estas a su vez favorecen las tormentas inflamatorias ${ }^{15-17}$.

Es importante considerar que México tiene uno de los más altos índices de enfermos con DM tipo 2 (DM2), con 12 millones de pacientes. Esta enfermedad es uno de tantos factores que predisponen y complican a la infección por SARS-CoV-2. Ante el incremento exponencial de dicha pandemia es importante tener en consideración todas las recomendaciones a la fecha para evitar el contagio y mantener las mejores condiciones de salud ante la posible infección. En el registro DISCOVER realizado en el país encontramos que el $50 \%$ de los enfermos con DM2 tienen hipertensión arterial sistémica, el $45.9 \%$ presentan elevación de cifras de colesterol vinculado a lipoproteínas de baja densidad (c-LDL), el $40.2 \%$ presentan obesidad y hasta un $13 \%$ padecen cardiopatía isquémica. Todas las comorbilidades son factores para el agravamiento por infección de SARS-CoV-2, por lo anterior, la población mexicana con DM2 es altamente susceptible de complicaciones, por lo que la mejor estrategia de protección es mantener un control adecuado de todos los factores de riesgo y considerar las siguientes recomendaciones fundamentales:

- La prescripción de alimentación debe ser supervisada por un personal de la salud, idealmente un nutriólogo, para individualizar los requerimientos de calorías, micronutrientes y macronutrientes.

- Dichos lineamientos están enfocados a disminuir los estados de malnutrición (desnutrición y obesidad), siendo más vulnerables los adultos mayores.

- Evitar al máximo la dieta típica occidental (rica en grasas saturadas, carbohidratos refinados y azúcares simples, bajos niveles de fibra, antioxidantes y grasas no saturadas).

- No comprar o empezar a consumir suplementos ni complementos alimenticios sin haber sido prescritos por un profesional de la salud.

- Moderar el consumo de sodio, alimentos procesados (industrializados) y de bebidas alcohólicas.

- En los pacientes infectados con enfermedad por coronavirus 2019 (COVID-19) y desnutridos los requerimientos son de 25 a $30 \mathrm{kcal} / \mathrm{kg}$ y $1.5 \mathrm{~g}$ de proteína/ $\mathrm{kg} / \mathrm{día}^{36}$.

- La adecuada suplementación de vitamina D se recomienda sobre todo en áreas endémicas que ya se conozcan con hipovitaminosis $D^{37}$.

- La presión arterial debe mantenerse, de acuerdo con las guías internacionales. Las europeas en 2018 como primera meta por debajo de $140 / 90 \mathrm{mmHg}$, puede elegirse una meta inferior de presión sistólica por abajo de 120-129 mmHg, en menores de 60 años; en mayores de 60 años puede elegirse una meta inferior a $130-139 \mathrm{mmHg}$, siempre analizando la relación entre riesgo y beneficio ${ }^{18}$. De acuerdo con 
las guías norteamericanas 2017 se recomienda una meta de presión menor a 130/80 $\mathrm{mmHg}^{19}$.

- El nivel de c-LDL idealmente llevarlo a $\leq 70 \mathrm{mg} / \mathrm{dl}$ y en caso de enfermedad cardiovascular por debajo de $55 \mathrm{mg} / \mathrm{dl}$, o bien considerar las guías norteamericanas American College of Cardiology/American Heart Association, que recomiendan una reducción de al menos el $50 \%$ del c-LDL basal para todo paciente diabético con y sin enfermedad cardiovascular establecida $^{20}$.

- La recomendación de la Asociación Americana de Diabetes 2020 sugiere mantener la glucosa en ayuno entre 80 y $130 \mathrm{mg} / \mathrm{dl}$ y es recomendable tener entre 140 y $180 \mathrm{mg} / \mathrm{dl}$ dos horas después de comer. En los pacientes que usan monitoreo continuo, es recomendable mantener tiempo en rango mayores a un $70 \%$ de las mediciones, es decir, entre 70 y $180 \mathrm{mg} / \mathrm{dl}$. De ahí la importancia de tener un plan de alimentación mucho más estricto en casa, sobre todo ahora, en tiempos de aislamiento social, lo que permitirá dentro de lo posible evitar episodios de hipoglucemia.

- Para adultos mayores con manifestación de SARS-CoV-2 leve a moderada o en aquellos que están usando esteroides con cifras de glucemia en ayuno de 110 a $140 \mathrm{mg} / \mathrm{dl}$ y posprandial $\leq 180 \mathrm{mg} / \mathrm{dl}$, debe valorarse el tratamiento actual para la DM con base en riesgos y advertencias a los pacientes. Para casos hospitalizados con manifestaciones moderadas de SARS-CoV-2, el régimen de insulina basal en bolos es lo más recomendable; en aquellos pacientes que se encuentran graves, se debe preferir el manejo con insulina por vía intravenosa.

- Para una persona con DM2 controlada y que no está bajo tratamiento con insulina, se recomienda revisar los niveles de glucosa capilar en ayuno y posprandial dos o tres veces por semana; si el resultado es $\geq 250 \mathrm{mg} / \mathrm{dl}$, se requiere consultar a su médico para valorar ajustes al tratamiento.

- Evitar consulta médica presencial a menos que sea estrictamente necesario y una vez agotado el recurso de consulta a distancia por videollamada o por Ilamada telefónica. Enfatizar que a la cita presencial se debe acudir solo con un acompañante y adherirse en todo momento al uso de mascarilla (cubriendo nariz, boca y mentón) y respetar el distanciamiento social. No saludar de mano ni tocar las superficies de los consultorios.

- Es indispensable que todos los médicos sugieran a los pacientes que tengan suficiente cantidad de medicamento y a los que requieren insulina, proveer de suficiente insulina para que no tengan que realizar visitas a las farmacias de manera frecuente. Así mismo, tener suficiente cantidad de tiras reactivas para glucómetros capilares y/o sensores para determinación del nivel de glucosa.

- Reforzar en los pacientes el impacto en su alimentación sana y mantener el aislamiento social; la transgresión de estas recomendaciones puede derivar en el descontrol de la glucosa y genera internamientos innecesarios

- Promover el ejercicio físico y el incremento de la actividad física en casa. Se pueden usar recursos como programas audiovisuales y de equipos de ejercicio doméstico, siempre bajo la autorización del personal médico.

- Mantener en todo momento el control de todos sus factores de riesgo, esto implica continuar su tratamiento como ha sido diseñado.

- De acuerdo con las recomendaciones ya ampliamente difundidas por diferentes sociedades médicas nacionales e internacionales, los pacientes con DM2 e hipertensión arterial sistémica que estén tomando medicamentos tipo inhibidor de la ECA (ramipril, lisinopril, enalapril, captopril, perindopril, etc.) o antagonistas de los receptores de la angiotensina II (ARA II) (losartán, valsartán, candesartán, azilsartán, telmisartán, etc.) no deben suspenderlos por ningún motivo a menos que su médico lo indique. No existe ninguna indicación en este momento para suspender o no iniciar el tratamiento con este grupo de fármacos ${ }^{21}$.

- Los medicamentos para la reducción del colesterol como las estatinas (atorvastatina, rosuvastatina, simvastatina, pitavastatina, etc.) y la ezetimiba deben seguir con la misma dosis indicada. No se tiene ninguna restricción en su uso, cuando han sido recetados por su médico. Lo mismo aplica para el uso de anticuerpos monoclonales que actúan selectivamente sobre la PCSK9 (evolocumab y alirocumab).

- En caso de estar tomando antiagregantes plaquetarios como aspirina, clopidogrel, ticagrelor o prasugrel, se debe continuar el tratamiento. En caso de alguna duda 0 inquietud en la dosis 0 en efectos adversos del medicamento, comunicarse con su médico para resolver dudas de manera conjunta.

- Los anticoagulantes orales antagonistas de la vitamina $\mathrm{K}$ (AVK) como la warfarina y la acenocumarina, o los anticoagulantes directos como el apixabán, el rivaroxabán o el dabigatrán no deben suspenderse y se deben continuar con las mismas dosis prescritas, sin dejar de tener comunicación con su médico, 
sobre todo si tuviera algún efecto adverso o para el ajuste de dosis, particularmente con los AVK ${ }^{22}$.

- Todo tratamiento tanto por vía oral como parenteral debe seguirse según indicación del médico. Hasta el momento, no existe ninguna evidencia para cambiar la conducta terapéutica. También se sabe que los pacientes que se encuentran mejor controlados de sus factores de riesgo tienen una menor posibilidad de agravarse en caso de contraer SARS-CoV-2.

- Se debe cuidar de manera muy estricta el estado de hidratación, evitar ayunos prolongados y el consumo excesivo de bebidas embriagantes.

- En los pacientes que padecen DM2 y que tienen múltiples factores de riesgo cardiovascular o que tienen enfermedad cardiovascular establecida, con insuficiencia cardiaca o con enfermedad renal grado leve o moderada, se recomienda que además de metformina y de no existir contraindicación, se pueden continuar o prescribir medicamentos de la familia de los inhibidores del cotransportador sodio-glucosa tipo 2 (iSGLT-2) y análogos del receptor del péptido 1 similar al glucagón (GLP-1). Sin embargo, en caso de hospitalización y por el riesgo de cetoacidosis en pacientes con SARS-CoV-2, se debe considerar descontinuarlos, sobre todo si existen eventos previos de cetoacidosis o en pacientes que reciben insulina. No se recomienda iniciar estos fármacos en pacientes ingresados con SARS-CoV-2 y que no los recibían previamente.

- Si se presentan signos y síntomas sugestivos de enfermedad por SARS-CoV-2 se recomienda llamar al 8000044800 o acudir a un hospital si los síntomas son graves.

- Es fundamental insistir en mantener una estrecha comunicación entre el médico y el paciente para ayudarlo y orientarlo; tratando, como se ha insistido, de utilizar herramientas no presenciales en lo posible.

- Si aparecen síntomas graves o dolor en el pecho, súbito o progresivo, se debe acudir a un servicio de urgencias $^{23}$.

\section{Aspectos fisiopatológicos}

Está establecido el vínculo entre hiperglucemia y SARS-CoV-2 ${ }^{24,25}$. Estudios in vitro muestran que el coronavirus se une a células blanco a través de la ECA 2, la cual se expresa en células epiteliales del pulmón, intestino, riñones y vasos sanguíneos. Es importante recordar que la expresión de la ECA 2 está incrementada en pacientes con diabetes, especialmente en aquellos que reciben un $\mathrm{IECA}$ o un ARA II, así como en pacientes que reciben tiazolidinedionas e ibuprofeno. Consecuentemente, en teoría la sobreexpresión de ECA 2 puede favorecer la infección severa por SARS-CoV-2. Esta información causó alarma en el mundo ante el número de pacientes que reciben estos fármacos, pero la rápida intervención de las sociedades científicas contuvo el cambio de tratamiento en los pacientes. En general, las personas con DM son susceptibles a complicaciones cuando son infectadas por SARS-CoV-2. La DM2 se asocia con un proceso inflamatorio crónico inducido por el tejido adiposo visceral, esto afecta a la regulación de la glucosa, así como a la sensibilidad periférica a la insulina. Estos factores (hiperglucemia e inflamación) favorecen una respuesta inmunitaria anormal e insuficiente (disminución en la movilización de polimorfonucleares, quimiotaxis, actividad fagocítica, baja producción de citocinas antiinflamatorias, aumento en la producción de IL-6 y glicación de inmunoglobulinas), lo que puede explicar el incremento de complicaciones en pacientes con diabetes y SARS-CoV-2. Sin embargo, resulta interesante cómo algunos fármacos hipoglucemiantes pueden (por lo menos en forma teórica) tener implicaciones en el pronóstico de los enfermos.

\section{Sulfonilureas}

Las sulfonilureas aumentan el riesgo de hipoglucemia y es mejor evitarlas en sujetos hospitalizados con enfermedades médicas graves.

\section{Metformina}

En su caso la metformina tiene acciones antiinflamatorias en estudios preclínicos y reduce los biomarcadores circulantes de inflamación en personas con DM2 ${ }^{35}$. También ha sido usada en pacientes no hospitalizados con otras infecciones virales, pero no en casos por SARS-CoV-2, por lo que debe usarse con precaución en pacientes hospitalizados inestables y debe suspenderse en personas con sepsis o insuficiencia severa de la función hepática y/o renal. Así mismo, es importante tener presente que se puede provocar deshidratación y acidosis láctica, particularmente si el paciente se encuentra previamente deshidratado, por lo que en estos casos se recomienda suspenderla ${ }^{26}$.

\section{Tiazolidinedionas}

El acúmulo de conocimiento señala que en las formas graves de infección por SARS-CoV-2 existe una 
liberación importante de mediadores proinflamatorios (entre los que predominan las IL 2, 6, 7 y 10, el factor estimulante de colonias de granulocitos, la proteína inducible 10, la proteína quimioatrayente de monocitos 1, la proteína 1 alfa inflamatoria de macrófago y el factor de necrosis tumoral alfa [TNF- $\alpha]$ ), desarrollando una manifestación de hiperinflamación sistémica. Existe la hipótesis de que la pioglitazona, perteneciente a la familia de tiazolidinedionas y administrada comúnmente con excelentes resultados para la resistencia a la insulina (donde existe un problema fisiopatológico proinflamatorio persistente), podría potencialmente ser administrada para reducir el proceso inflamatorio asociado al SARS-CoV-2, al menos en los pacientes con síndrome metabólico ${ }^{23}$.

Estudios experimentales ${ }^{24,25}$ analizaron el comportamiento de lesiones pulmonares inducidas y su evolución cuando se agrega pioglitazona al manejo. Los resultados demostraron una reducción significativamente en los niveles séricos de IL, TNF y el grado de lesión pulmonar inducido por sepsis. Al igual que otros fármacos, el papel de las tiazolidinedionas en el manejo de pacientes con hiperglucemia y SARS-CoV-2 es complejo y controvertido. Debemos esperar y observar el comportamiento del padecimiento en este grupo de personas que ya reciben estos medicamentos.

\section{Dipeptidil peptidasa 4}

La dipeptidil peptidasa 4 (DPP-4) humana fue identificada como un receptor funcional para la proteína espiga del coronavirus involucrado en el síndrome respiratorio de Oriente Medio (MERS-CoV). Este coronavirus se une al receptor de DPP-4 e interactúa con las células $T$ y el factor nuclear potenciador de las cadenas ligeras kappa de las células B activadas, que están implicados en la patogénesis de procesos inflamatorios. Interesantemente, se ha observado que los anticuerpos dirigidos contra DPP-4 inhiben la infección de células del epitelio bronquial causada por el hCoVEMC (human coronavirus-Erasmus Medical Center). La enzima DPP-4 es una glucoproteína transmembrana que se expresa en varios tejidos, incluyendo las células inmunitarias. Participa en el metabolismo de la glucosa y la insulina al degradar las incretinas y reducir la liberación de insulina. La expresión de DPP-4 es mayor en el tejido visceral y se relaciona con el proceso inflamatorio crónico, la resistencia a la insulina y la desregulación del sistema inmunitario. Si bien algunos estudios han demostrado que la administración de inhibidores DPP-4 reducen la posibilidad de infecciones respiratorias, los efectos inmunorreguladores de los inhibidores de la DPP-4 aún no son bien conocidos y se requieren más estudios para determinar si este efecto existe consistentemente. Entender la relación entre la DPP-4 y el SARS-CoV-2 puede ayudar a mejorar el tratamiento en este grupo tan tendente a complicarse a nivel sistémico. En individuos con infección activa por SARS-CoV-2 y depleción de volumen clínicamente significativa o sepsis sistémica, una reducción en la función renal puede requerir un ajuste de la dosis de algunos inhibidores de DPP-4, que incluyen alogliptina, linagliptina, sitagliptina y saxagliptina.

\section{Inhibidores del cotransportador sodio- glucosa tipo 2}

Los iSGLT-2, que incluyen canagliflozina, dapagliflozina y empagliflozina, son generalmente bien tolerados en el ámbito ambulatorio, además de ser cardioprotectores, especialmente en el contexto de la insuficiencia cardiaca con fracción de expulsión reducida, pero en el caso de la infección por SARS-CoV-2 (que puede estar asociada con anorexia, deshidratación y cetoacidosis diabética ${ }^{26}$, así como deterioro rápido del estado clínico) deben suspenderse de forma sistemática en pacientes inestables con infección grave por SARS-CoV-2 al ingreso en el hospital. Así mismo, en pacientes estables debe reevaluarse 0 descontinuarse ${ }^{37}$ y monitorizar cuidadosamente la lesión renal aguda cuando se sospeche deterioro renal.

\section{Agonistas del receptor del péptido 1 similar al glucagón}

Los agonistas del GLP-1 son hormonas secretadas por el intestino luego de la ingesta calórica. Se han explorado como agentes reductores de glucosa en el periodo perioperatorio y en la unidad de cuidados intensivos, y en general, se ha demostrado que son seguros y efectivos para el manejo de la glucosa en sangre $^{27}$. Sin embargo, el número total de sujetos estudiados es pequeño y la duración de la terapia es limitada. Aunque el GLP-1 reduce de manera segura la glucosa en sangre en estudios a corto plazo de pacientes ventilados con enfermedad crítica ${ }^{28}$, no hay experiencia suficiente en términos de la seguridad y el uso de agonistas del receptor del GLP-1 en sujetos críticos para hacer recomendaciones terapéuticas para el uso de estos agentes en el contexto de la infección por SARS-CoV-2 ${ }^{29}$, y las formulaciones basadas en 
exenatida deben detenerse en sujetos con deterioro de la función renal.

Se ha demostrado que la liraglutida es segura y efectiva cuando se usa para el control perioperatorio agudo de la glucosa en sangre en pacientes sometidos a cirugía cardiovascular electiva ${ }^{30}$. De manera similar, la exenatida parece segura y efectiva cuando se usa sola o en combinación con insulina basal para el manejo de la glucosa en sangre en pacientes hospitalizados no críticamente enfermos con DM2 tratados en medicina general o salas de cirugía ${ }^{31}$.

\section{Insulina}

La insulina se ha utilizado ampliamente durante décadas para controlar la glucosa en sujetos hospitalizados en estado crítico con diabetes e incluso puede reducir las tasas de hipoglucemia asociadas con el uso de insulina en el hospital en pacientes en unidades de terapia intensiva ${ }^{32}$. Además, la insulina ejerce acciones antiinflamatorias en humanos y reduce los biomarcadores de inflamación en individuos hospitalizados con enfermedades críticas $^{33}$. Entre los agentes disponibles para el tratamiento de enfermedades agudas complicadas por diabetes, la insulina ha sido el agente más utilizado en seres humanos con infecciones bacterianas o virales y en pacientes hospitalizados en estado crítico, sin embargo, hay poca información sobre los posibles beneficios o riesgos de la insulina en el contexto de la infección aguda por SARS-CoV-2. Se recomienda iniciar insulina intravenosa temprana en casos severos de síndrome de dificultad respiratoria aguda con titulación exacta para evitar la reabsorción subcutánea variable ${ }^{26}$.

En conclusión sabemos que las enfermedades crónicas, como las cardiovasculares y la diabetes, incrementan la morbimortalidad cuando se contagian de SARS-CoV-2, es por ello que se insiste en los cuidados generales, la alimentación y el control de cada una de las condiciones y comorbilidades con las que frecuentemente se asocian. Existe infinidad de publicaciones sobre terapéutica en dicha pandemia y se debe actuar con objetividad, análisis fisiopatológico del mecanismo de acción de los medicamentos y considerar que en la práctica regular en los pacientes externos es preferible utilizar la telemedicina y la comunicación estrecha con el médico tratante. Enfatizar la importancia de no suspender ningún tipo de medicamento y solamente en los casos de hospitalización se deberán individualizar algunos cambios terapéuticos, como ya se ha señalado.

\section{Responsabilidades éticas}

Protección de personas y animales. Los autores declaran que para esta investigación no se han realizado experimentos en seres humanos ni en animales.

Confidencialidad de los datos. Los autores declaran que han seguido los protocolos de su centro de trabajo sobre la publicación de datos de pacientes.

Derecho a la privacidad y consentimiento informado. Los autores han obtenido el consentimiento informado de los pacientes y/o sujetos referidos en el artículo. Este documento obra en poder del autor de correspondencia.

\section{Bibliografía}

1. Chen N, Zhou M, Dong X, Qu J, Gong F, Han Y, et al. Epidemiological and clinical characteristics of 99 cases of 2019 novel coronavirus pneumonia in Wuhan, China: a descriptive study. Lancet. 2020;395:507-13.

2. Wang D, Hu B, Hu C, Zhu F, Liu X, Zhang J, et al. Clinical characteristics of 138 hospitalized patients with 2019 novel coronavirus-infected pneumonia in Wuhan, China. JAMA. 2020; 323;1061-9.

3. Li B, Yang J, Zhao F, Zhi L, Wang X, Liu L, et al. Prevalence and impact of cardiovascular metabolic diseases on COVID-19 in China. Clin Res Cardiol. 2020;109(5):531-8.

4. Comprehensive Medical Evaluation and Assessment of Comorbidities: Standards of Medical Care in Diabetes-2020. American Diabetes Association. Diabetes Care. 2020; 43(Suppl 1): S37-S47.

5. Akbar DH. Bacterial pneumonia: comparison between diabetics and non-diabetics. Acta Diabet. 2001;38(2):77-82.

6. García Hernández RA, Rivero Seriel L, Aroche Aportela R Aldama Pérez LI, Hernández Navas M. COVID-19: en torno al sistema cardiovascular. Anales de la Academia de Ciencias de Cuba. 2020;10(2). Especial COVID-19.

7. Yang $X, Y u Y, X u J$, Shu $H$, Liu $H, W u Y$, et al. Clinical course and outcomes of critically ill patients with SARS-CoV-2 pneumonia in Wuhan, China: a single-centered, retrospective, observational study. Lancet Respir Med. 2020;8(5):475-81.

8. Rojas-Martínez R, Basto-Abreu A, Aguilar-Salinas CA, Zárate-Rojas E, Villalpando S, Barrientos-Gutiérrez T. Prevalencia de diabetes por diagnóstico médico previo en México. Salud Pública Méx. 2018:60:224-32.

9. Hill MA, Mantzoros C, Sowers JR. COVID-19 in patients with diabetes. Metabolism. 2020;107:154217.

10. Klonoff DC, Umpierrez GE. COVID-19 in patients with diabetes: risk factors that increase morbidity. Metabolism. 2020 Apr 7:154224. doi: 10.1016/j.metabol.2020.154224. [Epub ahead of print]

11. Wang W, Lu J, Gu W, Zhang Y, Liu J, Ning G. Care for diabetes with COVID-19: Advice from China. J Diabetes. J Diabetes. 2020;12(5):417-9.

12. Zhou F, Yu T, Du R, Fan G, Liu Y, Liu Z, et al.Clinical course and risk factors for mortality of adult inpatients with COVID-19 in Wuhan, China: a retrospective cohort study. Lancet. 2020;395(10229):1054-62.

13. Hussain A, Bhowmik B, do Vale Moreira NC. COVID-19 and diabetes: Knowledge in progress. Diabetes Res Clin Pract. 2020 Apr 9;162:108142. doi: 10.1016/j.diabres.2020.108142. [Epub ahead of print]

14. Vaninov N. In the eye of the COVID-19 cytokine storm. Nat Rev Immunol. 2020;20(5):277

15. Buonaguro FM, Ascierto PA, Buonaguro L, Morse GD, Tornesello ML, Puzanov I, et al. COVID-19: A paradigm change. (March 24, 2020). Available at SSRN: https://ssrn.com/abstract=3561224 or http://dx.doi. org/10.2139/ssrn.3561224

16. Chen $\mathrm{C}$, Zhang $X R$, Ju ZY, He WF. [Advances in the research of cytokine storm mechanism induced by Corona Virus Disease 2019 and the corresponding immunotherapies]. Zhonghua Shao Shang za zhi. 2020 Mar 1;36(0):E005. doi: 10.3760/cma.j.cn501120-20200224-00088. [Epub ahead of print]

17. Williams B, Mancia G, Spiering W, Agabiti Rosei E, Azizi M, Burnier M, et al. 2018 ESC/ESH Guidelines for the management of arterial hypertension. Eur Heart J. 2018;39:3021-104

18. Whelton PK, Carey RM, Aronow WS, Casey DE Jr, Collins KJ, Dennison Himmelfarb C, et al. 2017 ACC/AHA/AAPA/ABC/ACPM/AGS/APhA/ ASH/ASPC/NMA/PCNA Guideline for the Prevention, Detection, Evaluation, and Management of High Blood Pressure in Adults: A Report of the American College of Cardiology/American Heart Association Task 
Force on Clinical Practice Guidelines. J Am Coll Cardiol. 2018;71(19): e127-e248.

19. Grundy SM, Stone NJ, Bailey AL, Beam C, Birtcher KK, Blumenthal RS, et al. 2018 AHA/ACC/AACVPR/AAPA/ABC/ACPM/ADA/AGS/APhA ASPC/NLA/PCNA Guideline on the Management of Blood Cholesterol: Executive Summary: A Report of the American College of Cardiology/ American Heart Association Task Force on Clinical Practice Guidelines. J Am Coll Cardiol. 2019;73(24):3168-209.

20. Diabetes and Metabolic Syndrome: Clinical Research and Reviews. Gupta R, Ghosh A, Singh AK, Misra A. Clinical considerations for patients with diabetes in times of COVID-19 epidemic. 2020;14:e211-e212.

21. Canadian Diabetes Association. Appendix 8. Sick-day medication list. Can J Diabetes. 2018;42:S316.

22. American Diabetes Association. Introduction: Standards of Medical Care in Diabetes-2020. Diabetes Care. 2020;43(Suppl 1):S1-S2.

23. Carboni E, Carta AR, Carboni E.Can pioglitazone be potentially usefu therapeutically in treating patients with COVID-19? Med Hypotheses. 2020 Apr 22;140:109776. doi: 10.1016/j.mehy.2020.109776. [Epub ahead of print]

24. Kutsukake M, Matsutani T, Tamura K, Matsuda A, Kobayashi M, Tachikawa $\mathrm{E}$, et al. Pioglitazone attenuates lung injury by modulating adipose inflammation. J Surg Res. 2014;189(2):295-303.

25. Xie X, Sinha S, Yi Z, Langlais PR, Madan M, Bowen BP, et al. Role of adipocyte mitochondria in inflammation, lipemia and insulin sensitivity in humans: effects of pioglitazone treatment. Int J Obes (Lond). 2017 Aug 14 doi: 10.1038/ijo.2017.192. [Epub ahead of print]

26. Bornstein SR, Rubino F, Khunti K, Mingrone G, Hopkins D, Birkenfeld AL, et al. Practical recommendations for the management of diabetes in patients with COVID-19. Lancet Diabetes Endocrinol. 2020 Apr 23. doi: 10.1016/S2213-8587(20)30152-2. [Epub ahead of print]

27. Hulst AH, Plummer MP, Hollmann MW, DeVries JH, Preckel B, Deane $\mathrm{AM}$, et al. Systematic review of incretin therapy during peri-operative and intensive care. Crit Care. 2018;22(1):299.

28. Lee MY, Fraser JD, Chapman MJ, Sundararajan K, Umapathysivam MM Summers MJ, et al. The effect of exogenous glucose-dependent insuli- notropic polypeptide in combination with glucagon-like peptide-1 on glycemia in the critically ill. Diabetes Care. 2013;36:3333-6.

29. Pasquel FJ, Fayfman M, Umpierrez GE. Debate on insulin vs non-insulin use in the hospital setting-Is it time to revise the guidelines for the management of inpatient diabetes? Curr Diab Rep. 2019;19:65.

30. Hulst AH, Visscher MJ, Godfried MB, Thiel B, Gerritse BM, Scohy TV, et al. Liraglutide for perioperative management of hyperglycaemia in cardiac surgery patients: a multicenter randomized superiority trial. Diabetes Obes Metab. 2020;22:557-65.

31. Fayfman M, Galindo RJ, Rubin DJ, Mize DL, Anzola I, Urrutia MA, et al. A randomized controlled trial on the safety and efficacy of exenatide therapy for the inpatient management of general medicine and surgery patients with type 2 diabetes. Diabetes Care. 2019;42:450-6.

32. Lu M, Zuo Y, Guo J, Wen X, Kang Y. Continuous glucose monitoring system can improve the quality of glucose control and glucose variability compared with point-of-care measurement in critically ill patients: A randomized controlled trial. Medicine (Baltimore). 2018;97:e12138.

33. Hansen TK, Thiel S, Wouters PJ, Christiansen JS, van den Berghe G. Intensive insulin therapy exerts anti-inflammatory effects in critically ill patients and counteracts the adverse effect of low mannose-binding lectin levels. J Clin Endocrinol Metab. 2003;88(3):1082-8.

34. Hamblin PS, Wong R, Ekinci El, Fourlanos S, Shah S, Jones AR, et al. SGLT2 inhibitors increase the risk of diabetic ketoacidosis developing in the community and during hospital admission. J Clin Endocrinol Metab. 2019;104(8):3077-87.

35. Cameron AR, Morrison VL, Levin D, Mohan M, Forteath C, Beall C, et al. Anti-inflammatory effects of metformin irrespective of diabetes status. Circ Res. 2016;119(5):652-65.

36. Barazzoni R, Bischoff SC, Breda J, Wickramasinghe K, Krznaric Z, Nitzan D, et al. ESPEN expert statements and practical guidance for nutritional management of individuals with SARS-CoV-2 infection. Clin Nutr. 2020 Mar 31. doi: 10.1016/j.clnu.2020.03.022. [Epub ahead of print]

37. Giustina A, Adler RA, Binkley N, Bouillon R, Ebeling PR, Lazaretti-Castro $\mathrm{M}$, et al. Controversies in vitamin $\mathrm{D}$ : summary statement from an international Conference. J Clin Endocrinol Metab. 2019;104(2):234-40. 